\title{
CORRESPONDENCE
}

\section{COVID-19 outbreak in children and/or adolescents}

@ The Author(s), under exclusive licence to the International Pediatric Research Foundation, Inc 2021

Pediatric Research (2022) 91:473; https://doi.org/10.1038/s41390021-01787-x

We would like to thank Cai et al. ${ }^{1}$ for your interest regarding our 2020 meta-analysis ${ }^{2}$ on the overall prevalence of clinical signs, symptoms, and radiological findings in children and/or adolescents with coronavirus disease 2019 (COVID-19). We offer the following response to the issues raised. First, Cai et al. ${ }^{1}$ wrote that we had to exclude the references \#21, \#27, and \#28, as these manuscripts were not published in English. Although these references were not published in English, we selected to include them, as their English abstracts provided all the data needed for our meta-analysis. In this context, it is also important to consider the period in which our meta-analysis ${ }^{2}$ was carried out. In the first months of 2020, indeed, any information on COVID-19 in children and/or adolescents was relevant, and, hence, we considered appropriate to use the works in which the abstracts in English allowed us to obtain useful information. The "non-English" works we have excluded referred to those completely written in Chinese or other languages, from which we were unable to extrapolate information to carry out a meta-analysis. This may be a bias that we have recognized in the work. ${ }^{2}$ Second, they wrote that we failed to exclude studies suspected of including duplicate reporting. Indeed, we did our best to extract information regarding the hospitals and recruitment periods of all eligible studies, in order to reduce the overlapping risk. However, given that no authors of the eligible studies in our meta-analysis clearly specified whether patients in their published manuscripts had been reported in other publications, we might have failed to remove the overlapping risk completely. ${ }^{3}$ That said, this issue may be present in most (if not all) of the studies on COVID-19. ${ }^{3}$

We believe that our meta-analysis had been important and useful for pediatricians and had provided important information regarding COVID-19 outbreak in children and/or adolescents. In addition, the message of our meta-analysis has been confirmed by other relevant studies. ${ }^{4,5}$ However, the clinical scenario of COVID-19 outbreak is constantly evolving and it is reasonable to think that these data should be updated to the current state.

Alessandro Mantovani ${ }^{1 凶}$, Elisabetta Rinaldi ${ }^{1}$, Chiara Zusi ${ }^{1,2}$, Giorgia Beatrice ${ }^{1}$, Marco Deganello Saccomani ${ }^{3}$ and Andrea Dalbeni ${ }^{4}$

${ }^{1}$ Section of Endocrinology, Diabetes and Metabolism, Department of Medicine, University and Azienda Ospedaliera Universitaria Integrata of Verona, Verona, Italy.

${ }^{2}$ Pediatric Diabetes and Metabolic Disorders Unit, Department of Surgical Sciences, Dentistry, and Pediatrics, and Gynaecology,
University Hospital of Verona, Verona, Italy. ${ }^{3}$ Department of Pediatrics, Woman's and Child's University Hospital of Verona, Verona, Italy. ${ }^{4}$ Section of General Medicine, Hypertension and Liver Unit, University and Azienda Ospedaliera Universitaria Integrata of Verona, Verona, Italy. ${ }^{\circledR e m a i l: ~ a l e s s a n d r o . m a n t o v a n i @ u n i v r . i t ~}$

\section{REFERENCES}

1. Cai, J., Lin, Y. \& Wu, M. J. More on SARS-COV-2 infection in children and adolescents. Pediatr. Res. https://doi.org/10.1038/s41390-021-01723-z (2021).

2. Mantovani, A. et al. Coronavirus disease 2019 (COVID-19) in children and/or adolescents: a meta-analysis. Pediatr. Res. 89, 733-737 (2021).

3. Bauchner, H., Golub, R. M. \& Zylke, J. Editorial Concern-Possible reporting of the same patients with COVID-19 in different reports. JAMA 323, 1256 (2020).

4. Cui, X. et al. A systematic review and meta-analysis of children with coronavirus disease 2019 (COVID-19). J. Med. Virol. 93, 1057-1069 (2021).

5. Castagnoli, R. et al. Severe acute respiratory syndrome coronavirus 2 (SARS-CoV-2) infection in children and adolescents: a systematic review. JAMA Pediatr. 174, 882-889 (2020).

\section{AUTHOR CONTRIBUTIONS}

All authors approved the final manuscript as submitted and agree to be accountable for all aspects of the work.

\section{FUNDING INFORMATION}

None.

\section{COMPETING INTERESTS}

The authors declare no competing interests.

\section{ETHICS APPROVAL AND CONSENT TO PARTICIPATE}

Not required.

\section{ADDITIONAL INFORMATION}

Correspondence and requests for materials should be addressed to Alessandro Mantovani.

Reprints and permission information is available at http://www.nature.com/ reprints

Publisher's note Springer Nature remains neutral with regard to jurisdictional claims in published maps and institutional affiliations. 